\title{
In -vitro Biofilm Formation of Vaginal Isolates of Streptococcus agalactiae; Effect of $\mathrm{pH}$ and Culture Media
}

Guruge Niluka Dilrukshi ${ }^{1,2}$

https://orcid.org/0000-0003-2167-6686

Jananie Kottahachchi ${ }^{1^{*}}$

https://orcid.org/ 0000-0002-0321-3250

Thushari Dissanayake ${ }^{1}$

https://orcid.org/0000-0002-2843-9651

Manjula Weerasekera ${ }^{1}$

https://orcid.org/0000-0002-8194-2205
Mudara Peiris ${ }^{1}$

https://orcid.org/0000-0002-4858-0185

Sohani Medis ${ }^{1}$

https://orcid.org/ 0000-0002-2984-6996

Neluka Fernando ${ }^{1}$

https://orcid.org/0000-0001-7551-8224

1 University of Sri Jayewardenepura, Faculty of Medical Sciences, Department of Microbiology, Nugegoda, Sri Lanka 1;

${ }^{2}$ Medical Research Institute, Colombo, Sri Lanka 2.

Editor-in-Chief: Alexandre Rasi Aoki

Associate Editor: Renata Marino Romano

Received: 2021.03.21; Accepted: 2021.06.27.

*Correspondence: jananie@sjp.ac.lk, jananiekottahachchi@yahoo.com; Tel.: +94772987942 (J.K.)

\section{HIGHLIGHTS}

- Maximum biofilm formation of GBS is at neutral $\mathrm{pH}(\mathrm{pH} 7)$.

- At a $\mathrm{pH}$ of 4.5 , there is a greater reduction in viable cells than at other $\mathrm{pH}$ value.

- At Normal vaginal pH (3.5 - 4.5), biofilm formation by GBS is low.

- Glucose concentration in the media influences biofilm production.

- Nutrient rich culture media support biofilm growth.

Abstract: Background: Streptococcus agalactiae (GBS), a major cause of neonatal morbidity and mortality, is transmitted from mother to neonate via placenta or during birth. Biofilm formation is an important factor in GBS pathogenesis. This study aimed to determine effects of $\mathrm{pH}$, different culture media and nutritional composition on in vitro biofilm forming ability of GBS isolated from pregnant women. Methods: A total of 30 confirmed isolates of GBS from pregnant women were tested for biofilm formation in Todd Hewitt Broth (THB) at $\mathrm{pH} 4.5,6$ and 7 . Ten of these isolates were tested for biofilm formation in growth media THB, brain heart infusion broth, tryptic soy broth, Mueller Hinton broth and nutrient broth. Further they were tested for influence of glucose on biofilm formation using crystal violet and MTT assay. Results: Of 30 GBS isolates strong biofilm formation (SBF) was observed at $\mathrm{pH} 7$ in $56.6 \%(\mathrm{n}=17)$ while $36.6 \%(\mathrm{n}=11)$ isolates showed weak biofilm formation (WBF). At pH 4.5, 43.3\% $(n=13)$ were non biofilm formers. In THB without glucose, all 10 isolates were SBF while THB with $1 \%$ glucose, $3(30 \%)$ isolates were SBF, $5(50 \%)$ isolates were moderate biofilm producers and $2(20 \%)$ isolates were WBF. Ten isolates tested in 5 types of growth media did not show statistically significant difference in biofilm forming ability. Conclusion: All tested vaginal GBS isolates were 
able to produce biofilms, maximum biofilm formation of GBS was at $\mathrm{pH}$ 7.0. and $\mathrm{pH} 4.5$ is not favorable, thus in normal vaginal $\mathrm{pH}(3.5-4.5)$, GBS finds it difficult to grow biofilms.

Keywords: Group B Streptococcus 1; Biofilm 2; Vaginal pH 3; Growth media 4.

\section{INTRODUCTION}

Streptococcus agalactiae (Group B Streptococcus, GBS) is a Gram-positive chain forming cocci and an inhabitant in normal flora in the gastro-intestinal tract and the vagina of healthy women. It causes neonatal sepsis, meningitis and pneumonia and leads to neonatal morbidity and mortality by reaching the newborn through the birth canal before or during birth. Global mean incidence of early onset and late onset neonatal infection is 0.53 cases of GBS per 1000 live births [1]. It causes adverse effects in pregnancy and also could result in bacteremia, skin and soft tissue infections, pneumonia, osteomyelitis and urinary tract infections in the elderly and in immuno-compromised people [2].

Highly enriched liquid or solid media are used to cultivate GBS. However, bacteria show entirely different behavior in-vivo than in vitro [3]. Sessile bacteria grown on nutrient-limited media show slow growth while planktonic bacteria multiply rapidly $[3,4]$. Therefore, planktonic bacteria are more susceptible to antibiotics, environmental and host factors compared to its sessile biofilm [3]. Colonization and disease progression of bacteria is linked with biofilm formation and it is an important virulence determinant. Biofilms facilitate chronic infections and antibiotic resistance. Bacteria are protected against the host immune system by biofilms. Consequently, these biofilm forming strains could be responsible for recurrent infections. Further biofilm like structure production could be a virulence factor in the role of pathogenesis of GBS in pregnant women and neonatal infection [5-7]. The presence of structures of the extracellular matrix typical of bacterial biofilms has not been investigated in GBS. Therefore, additional studies are also needed to confirm the in-vitro biofilm formation ability of GBS.

$\mathrm{pH}$ of the host environment fluctuates from neutral $\mathrm{pH}$ to acidic $\mathrm{pH}$ of the vagina. GBS strains should be able to adapt to this range as a colonizer of the vagina. Normal vaginal $\mathrm{pH}$ of women varies $3.5-4.5$. Lactobacillus spp. produce lactic acid from glycogen and maintain the acidic $\mathrm{pH}$ of the vagina [8]. Some studies concluded that the biofilm formation of GBS is $\mathrm{pH}$ dependent and bacterial biofilm formation varies due to $\mathrm{pH}$ and the growth medium [6]. Some studies investigated GBS biofilm formation under neutral and acidic $\mathrm{pH}$ conditions and found that higher biofilm formation occurred at $\mathrm{pH} 6.5$ or 7 than normal vaginal $\mathrm{pH}$ 4.2. However, Ho et al found that biofilm formation was enhanced at low pH, 4.2 [9]. Conflicting data have been reported regarding biofilm forming ability at different $\mathrm{pHs}$.

Under in- vitro conditions, the composition of the culture medium is an important factor to produce biofilm $[10,11]$. Todd Hewitt broth (THB) is useful for cultivating GBS since it is a highly nutritious medium and the $\mathrm{pH}$ of the medium is $7.8 \pm 0.3$. Further, it is reported to be the most commonly used medium to study biofilm forming ability of GBS. Nutrition component of the brain heart infusion broth (BHI) is almost similar to THB except the $\mathrm{pH}(\mathrm{pH} 7.4 \pm 0.2)$. Other media used for the cultivation of microorganism include, Muller Hinton broth (MHB), Tryptic soy broth (TSB) and nutrient broth (NB). One study found that the biofilm formation was induced in nutrient limited medium than in rich media [6] and also Konto-Ghiorghi et al. concluded that biofilm formation was reduced in nutrient rich media by GBS serotype III [12]. It is observed that presence of glucose or sucrose in the THB induces biofilm formation of GBS serotype la [13].

Relatively few studies have been published on GBS biofilm formation and factors affecting such as $\mathrm{pH}$ level of the medium, influence of different growth media and glucose for biofilm formation of GBS. Additional studies are needed to confirm the biofilm formation of GBS, and optimum conditions relevant to biofilm formation in vitro. In order to determine biofilm formation ability artificially, it is essential to identify and standardize different growth media components and nutritional conditions for biofilm formation of GBS. Earlier recommendations of appropriate growth medium for testing of GBS biofilm formation yielded controversial results, thus this study aimed to determine a suitable $\mathrm{pH}$ and medium. Considering above facts this study was formulated to satisfy the gaps in knowledge on biofilm formation by GBS.

\section{MATERIAL AND METHODS}

\section{Test Strains}

In this study a total of 30 confirmed isolates of GBS yielded from vaginal swabs from pregnant women at $>35$ weeks of gestation were tested for biofilm forming ability. The isolates were initially identified by 
standard microbiological methods including characteristic colonies with beta haemolysis, Gram-positive cocci in chains (by Gram stain), negative catalase test, negative reaction in bile aesculin agar, positive by ChristieAtkins-Munch-Petersen (CAMP) test and detection of capsular polysaccharide antigen by latex agglutination (Streptococcal Lanced field grouping kit, Oxoid, UK). Isolates were stored in skimmed milk at $-80^{\circ} \mathrm{C}$.

\section{Ethical approval}

Ethical approval was obtained from the ethics review committee of the Faculty of Medical Sciences, University of Sri Jayewardenepura, Sri Lanka (No.89/17).

\section{Biofilm forming ability}

Colonies of 24 hour old cultures grown on blood agar were inoculated separately in THB and incubated at $37^{\circ} \mathrm{C}$ overnight. After incubation cell pellets were obtained by centrifugation at $3000 \mathrm{rpm}$ for $10 \mathrm{~min}$. Cell pellets were washed twice with sterile phosphate buffered saline (PBS). Standard inoculum of each GBS strains were adjusted to the optical density (OD) 0.1 at $595 \mathrm{~nm}$ wavelength ((Multiskan EX, Thermo Scientific, USA) (0.1 OD corresponds to $1 \times 10^{8}$ cells $\left./ \mathrm{mL}\right)$ ) and $200 \mu \mathrm{L}$ of standard cell suspensions were added into each well. The plates were sealed and incubated at $37^{\circ} \mathrm{C}$ for 24 hours in sterile flat bottomed 96 -wells microtiter plates [6]. Following incubation, the medium was removed carefully and the wells were washed three times with $200 \mu \mathrm{L}$ of sterile phosphate buffered saline (PBS) to remove unattached bacteria. Crystal violet (CV) and tetrazolium salt 3-[4, 5-dimethylthiazol-2-yl]-2, 5-diphenyltetrazolium bromide (MTT) assays were then performed to quantify bacterial biomass and cell viability, respectively. A known biofilm former, Staphylococcus aureus (ATCC 25923) was used as positive control. The experiment was carried out in triplicate with three independent experiments to maintain the reproducibility [14,]

\section{Effect of pH changes on S. agalactiae biofilm}

THB acidified to $\mathrm{pH} 4.5,6$ and 7. $\mathrm{pH}$ was adjusted in the class II bio safety cabinet near the flame. THB was prepared and poured in to 3 sterile universal containers (labeled as $\mathrm{pH} 4.5,6.0$ and 7.0). Initial $\mathrm{pH}$ of THB was 8.2. Few drops of concentration $\mathrm{HCl}$ was added separately into 3 bottles by using sterile pasture pipette. Small portion of media was taken in to 3 sterile containers separately and $\mathrm{pH}$ was measured by $\mathrm{pH}$ meter. This procedure was repeated until it reached the correct $\mathrm{pH}$ values. Biofilm formation was assessed as described. The $\mathrm{pH}$ value of 4.5 was used to reproduce the normal vaginal $\mathrm{pH}$ and $\mathrm{pH} 6$ and 7 were taken as representative of abnormal vaginal $\mathrm{pH}$.

\section{Influence of glucose on S. agalactiae biofilm}

Ten S. agalactiae isolates were tested for their biofilm forming ability with THB and THB supplemented with $1 \%$ glucose. One gram of glucose was measured and dissolved in THB. The solution was filtered by Seitz filter in to sterile container and final volume was adjusted up to $100 \mathrm{ml}$ with THB. Biofilm formation was detected as mentioned above.

\section{Effect of growth media on biofilm formation}

Biofilm forming ability of 10 GBS isolates were further tested on Todd Hewitt Broth (Oxoid), brain heart infusion broth (Oxoid), tryptic soy broth (Oxoid), Mueller Hinton broth (Oxoid) and nutrient broth (Oxoid).

Five types of broth media were prepared to standard protocol. Colonies of 24 hour old cultures grown on blood agar were inoculated separately in five types of growth media and incubated at $37^{\circ} \mathrm{C}$ overnight.

Standard inoculum of each GBS strains were adjusted to the optical density (OD) 0.1 at $595 \mathrm{~nm}$ wavelength in 5 types of growth media separately. Biofilm formation was detected as described.

\section{Crystal Violet assay}

Crystal violet (CV) assay was performed as follows. The wells were stained for 10 min with $200 \mu \mathrm{l}$ of a $0.5 \%$ solution of CV. After rinsing with distilled water, bound dye in stained cells was eluted with $200 \mu \mathrm{l}$ of $30 \%$ glacial acetic acid. Biofilm formation was quantified by measuring the OD595 of the solution with a microplate reader assay. Wells filled only with growth medium were included as negative controls. OD of stained bacterial films were measured at $595 \mathrm{~nm}$ using ELISA plate reader (Multiskan EX, Thermo Scientific, USA) 
$\mathrm{OD}$ (isolate) $<\mathrm{OD}$ (cut) $=$ non-biofilm-producing

OD (cut) $<$ OD (isolate) $<2 O D$ (cut) = weak-producing

$2 O D$ (cut) $<$ OD (isolate) $<4 O D$ (cut) = moderate-producing

$4 O D$ (cut) $<$ OD (isolate) $=$ strong-producing

were categorized compared with the negative control (culture medium only). (ODcut $=$ ODavg of negative control $+3 \times$ standard deviation of ODs of negative control). $[14,15]$.

(OD (cut) - Optical density cut-off value, OD (isolate) - Optical density isolate, ODavg - Optical density average).

\section{MTT viability assay}

MTT (tetrazolium salt 3-[4, 5-dimethylthiazol-2-yl]-2, 5-diphenyltetrazolium bromide; Sigma-Aldrich) assay [14,15], was done as follows. The working solution of $1 \mathrm{mg} / \mathrm{mL}$ MTT was prepared by dissolving in sterile distilled water and was filtered. Viable cell mass was quantified by adding $50 \mu \mathrm{L}$ of working solution in to each well and incubated at $37^{\circ} \mathrm{C}$ for $4 \mathrm{~h}$. The micro titer plates were then covered by aluminum foil. The remaining MTT solution was aspirated carefully after incubation and $100 \mu \mathrm{L}$ of dimethyl sulfoxide (SigmaAldrich) (DMSO) was added to each well to solubilize the black purple colour formazan end product. Absorbance was measured at 570 and $630 \mathrm{~nm}$ wavelengths. [16].

\section{Statistical analysis}

The data were analyzed using the statistical package GraphPad Prism version 9 by one-way ANOVA and Microsoft Excel 2016. Values with a $p<0.05$ was considered as statistically significant.

\section{RESULTS}

\section{Effect of pH changes on biofilm formation}

Biofilm formation was assessed for the 30 isolates under varying $\mathrm{pH}$ conditions of $\mathrm{pH} 4.5,6$ and 7 by CV assay. Biofilm formation was higher with increasing $\mathrm{pH}$ in the growth medium. (Figure 1)

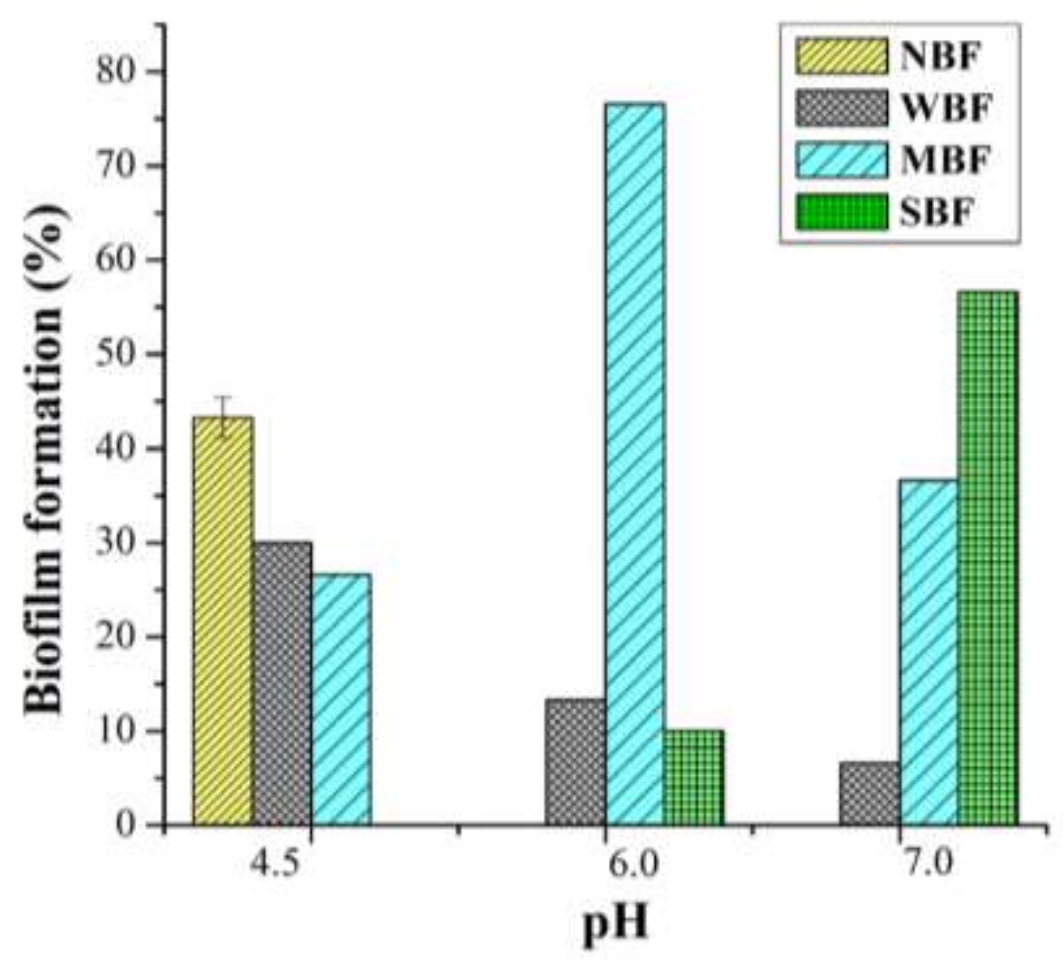

Figure 1. Percentage of Biofilm formation by GBS at different pH by CV assay

Legend: Thirty GBS strains isolated from pregnant women were grown in 96-well polystyrene plates in THB medium at $\mathrm{pH}$ levels 4.5, 6.0 and 7.0 for $24 \mathrm{~h}$. Biofilm formation was quantified by crystal violet assay.

At $\mathrm{pH} 7$, seventeen (56.6\%) GBS isolates gave OD more than 0.712 and were categorized as strong biofilm formers, while 11 (36.6\%) GBS isolates gave OD between 0.178 and 0.356 and were weak biofilm 
formers. The growth was reduced at $\mathrm{pH} 4.5$ and 13 (43.3\%) GBS isolates gave OD less than 0.178 and were non biofilm formers.

Biofilm formation was assessed for the 30 isolates under varying $\mathrm{pH}$ conditions of $\mathrm{pH} 4.5,6$ and 7 by MTT assay. (Table 1)

Table 1. Percentage of Biofilm formation by GBS at different $\mathrm{pH}$ by MTT assay.

$\mathrm{pH}$ value

Percentage of Biofilm formation by GBS

4.5

6.0

7.0

$$
\text { Strong } \mathrm{No}^{*}(\%)
$$

0

$5(16.6 \%)$ $13(43.3 \%)$

$$
\text { Moderate } \mathrm{No}^{*}(\%)
$$

$5(16.6 \%)$ $11(36.6 \%)$
Weak $\mathrm{No}^{*}(\%)$

$3(10 \%)$

$19(63.3 \%)$

$6(20 \%)$
Non biofilm No*(\%)

$27(90 \%)$

$1(3.3 \%)$

Legend: Thirty GBS strains isolated from pregnant women were grown in 96-well polystyrene plates in THB medium at $\mathrm{pH}$ levels 4.5, 6.0 and 7.0 for $24 \mathrm{~h}$. Biofilm formation was quantified by MTT assay. No* - Number of GBS bacterial isolates.

Strong biofilm formation (SBF) was observed at $\mathrm{pH}$ 7. Thirteen (43.3\%) GBS isolates gave OD more than 0.248 and were categorized as strong biofilm formers and $11(36.6 \%)$ GBS isolates gave OD between 0.248 and 0.124 and were moderate biofilm formers (MBF), however, growth was reduced at pH 4.5 with 27 $(90 \%)$ GBS isolates being non biofilm formers (NBF) giving OD value less than 0.062 .

\section{Influence of glucose on S. agalactiae biofilms}

Determination of the influence of addition of glucose on the ability of biofilm formation of 10 isolates of GBS was done. THB with and without $1 \%$ glucose was tested. In THB medium without glucose, $100 \%$ isolates were strong biofilm producers while THB with glucose $3(30 \%)$ isolates were strong biofilm producers, 5 $(50 \%)$ isolates were moderate biofilm producers and $2(20 \%)$ isolates were weak biofilm producers. (Figure 2)

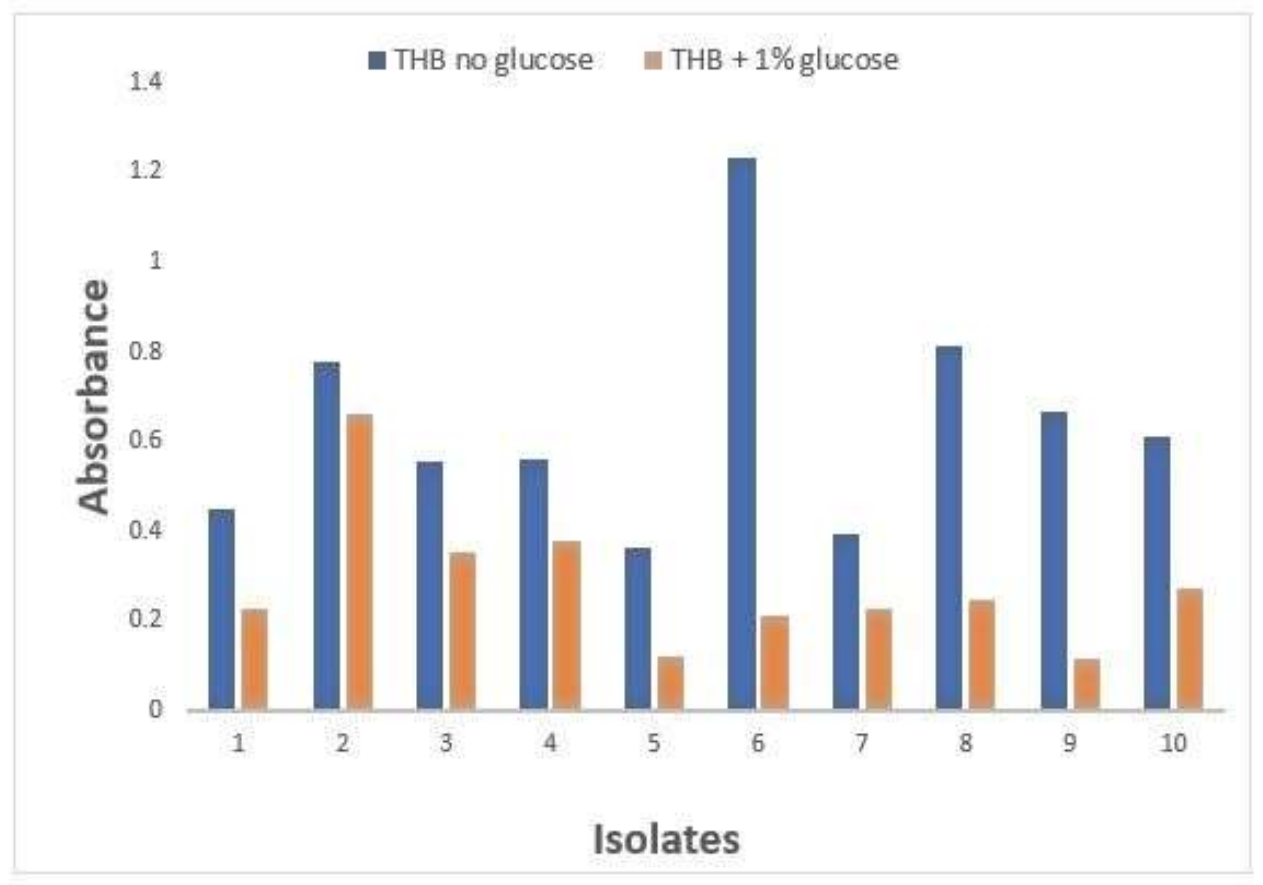

Figure 2. Influence of glucose on S. agalactiae biofilm

Legend: Ten GBS isolates were tested for their biofilm forming ability with THB and THB supplemented with $1 \%$ glucose by MTT assay. 


\section{Effect of growth media on biofilm formation}

Ten isolates were tested to detect biofilm formation in 5 types of growth media. OD values represent biofilm forming ability of ten isolates in different culture media. THB was used as control medium. TSB showed the least average OD value, therefore TSB medium supported GBS biofilm formation to the least extent, while the growth of biofilms of other four media were comparatively high. A one-way between groups analysis of covariance was conducted to compare the biofilm formation of GBS of five different culture media. The independent variable was the five types of culture media (BHI, TSB, NB, THB, MHB) and the dependent variable was optical density of biofilm of GBS after overnight incubation. Optical density of different culture media (without GBS) was used as the covariate. There was no significant difference between the five types of culture media on biofilm formation ability of GBS, indicating that growth of biofilm did not differ with the media used. (Figure 3).

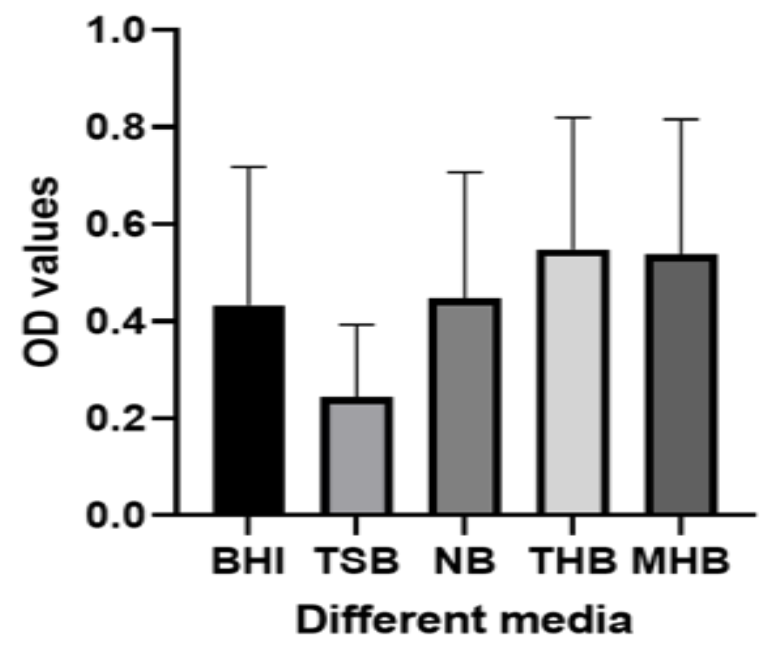

Figure 3. Effect of different growth media on GBS biofilm formation.

Legend: GBS biofilm formation in 5 different growth media: Brain Heart Infusion broth (BHI), Tryptic Soy Broth (TSB), Nutrient Broth (NB), Todd Hewitt Broth (THB), Mueller Hinton Broth (MHB). The error bars represent $\pm 2 \mathrm{SD}$.

\section{DISCUSSION}

Investigating the biofilm forming ability of GBS is important in determining the virulence of the pathogen [6]. Limited nutrients, variations of $\mathrm{pH}$, effects of disinfectants and antibiotics can be tolerated better by the biofilm producers than planktonic organisms [17]. Dominant microbiota of the healthy vagina are lactobacilli. They maintain the vaginal acidity by producing lactic acid [18]. Normal vaginal $\mathrm{pH}$ is around 4.5. Disrupting normal flora of the vagina increases the $\mathrm{pH}$ and is a favorable condition for the biofilm formation of pathogenic bacteria. Lactobacilli inhibit the colonization of bacterial pathogens by maintaining the vaginal acidity. They produce lactic acid, hydrogen peroxide, and bacteriocins which has antibacterial properties against vaginal pathogens including GBS [19,20]. A study reported that the presence of Lactobacillus was significantly lower whereas Gram negative bacteria and streptococci were significantly higher in GBS positive pregnant mothers [21]. GBS survival and biofilm production could be influenced by increased vaginal $\mathrm{pH}[18,20,22]$. This study observed that the maximum biofilm formation of GBS was at the neutral $\mathrm{pH}(\mathrm{pH} 7)$ and acidic $\mathrm{pH}$ is not favorable for growth of GBS. At a pH of 4.5, there was a greater reduction in viable cells than at other $\mathrm{pH}$ value, thus demonstrating that in normal vaginal $\mathrm{pH}, \mathrm{GBS}$ finds difficult to grow biofilms. These results are in agreement with Borges et al. (2011) who observed a reduction of viable cells at pH 4.2 than at pH 5.5 and 6.5 and further that GBS survived longer at higher $\mathrm{pH}$ than at normal vaginal $\mathrm{pH}$ [5]. $\mathrm{H}$. Kuar et al (2009) found that the biofilm formation was more sensitive to $\mathrm{pH}$ changes and decreased at $\mathrm{pH}$ below 6.0 [22]. Lamees A. Abdul-Lateef et al (2018) also concluded similar results to current study that the GBS isolates formed biofilms at $\mathrm{pH} 6.0$ and $\mathrm{pH} 5.0$ but not at $\mathrm{pH} 4.0$ [20]. Controversial findings were reported by Ho et al. (2013) who reported that biofilm formation of GBS induced at low pH in nutrient limited medium and not in THB which is a rich media [6]. Majority of the studies in keeping with the findings of the current study indicate that GBS biofilm forming ability is less in normal vaginal $\mathrm{pH}$. This finding is favorable for healthy women as the normal vaginal $\mathrm{pH}$ inhibits GBS biofilm formation. 
However, the presence of vaginal pathogens may inhibit lactobacilli in the vagina. Reduction of lactobacilli in the vagina results in change of the low $\mathrm{pH}$ to a high $\mathrm{pH}$. It is reported that GBS either as a colonizer and or a pathogen, could change the vaginal $\mathrm{pH}$ [23]. Increasing the $\mathrm{pH}$ level is favorable for biofilm formation of GBS. Enhanced biofilm formation is associated with a magnitude of issues such as antibiotic resistance.

Carbohydrate content, was found to influence streptococcal biofilm formation in vitro. Several studies concluded that the addition of glucose is necessary for the biofilm formation. Konto-Ghiorghi et al. found a uniform biofilm formation on Luria-Bertani (LB) and Roswell Park Memorial Institute Medium (RPMI 1640), supplemented with $1 \%$ glucose [12]. Rinaudo et al observed that addition of $1 \%$ glucose to THB increased the GBS biofilm formation [13]. By contrast, another study concluded that by adding various sugars to enrich the medium does not favor the biofilm formation of GBS and further it was observed that starvation conditions initiate biofilm formation [22]. In the present study we observed that addition of $1 \%$ glucose to THB decreased the biofilm formation ability and all the tested isolates formed strong biofilms in the absence of $1 \%$ glucose. Further in this study, the absence of supplemented $1 \%$ glucose in the basal media (THB) favored biofilm growth, however, it is not statistically significant. It is envisaged that the production of acid by the fermentation of glucose, decreases the $\mathrm{pH}$ of the medium. Therefore, the biofilm formation is less favorable in glucose rich medium due to low $\mathrm{pH}$ level as seen in this study.

The composition of growth medium affects biofilm formation of bacteria. Biofilm formation of some bacterial species such as Streptococcus gordonii and Enterococcus faecalis can be observed in nutrient poor media while Vibrio cholerae needs nutrient-rich media to form biofilm $[6,24]$.

In several studies TSB and BHI were the media of choice to detect the biofilm formation of bacteria $[20,22]$ however THB is the medium used most of the time to grow GBS biofilms $[6,25]$. However, Singh et al concluded that BHI broth was more effective for biofilm formation than TSB [26]. It was found that Staphylococcus epidermidis showed better biofilm formation in TSB than in MHB. Borges et al (2011) found that Listeria monocytogenes produces more biofilm in nutrient-rich media such as TSB than in nutrient-poor media and further concluded in 2012 that GBS biofilm formation ability was higher in TSB than simulated vaginal fluid $[5,8]$. However, Konto-Ghiorghi et al. demonstrated that biofilm formation by GBS serotype III was reduced in nutritionally rich media (THB) compared to weaker media (RPMI) [12]. In the present study we investigated GBS biofilm forming ability in 5 different media such as THB, BHI, TSB, MHB and NB. This study hypothesized that the other four growth media would have similar biofilm formation abilities by GBS when compared to growth medium of choice; THB.

According to findings of this study,all tested media facilitated GBS biofilm formation. These data to some extent confirms the findings of Borges et al. who showed that the high nutrient composition broth media could facilitate to produce biofilms. Although THB has been the choice of medium for GBS biofilm formation, in the absence of statistically significant difference of mean OD values of five different media, it can be inferred that any medium described could be used to detect biofilm of GBS.

\section{CONCLUSION}

All tested vaginal GBS isolates were able to produce biofilms, maximum biofilm formation of GBS was at neutral $\mathrm{pH}(\mathrm{pH} 7)$ and acidic $\mathrm{pH}$ was not favorable for growth of GBS. At $\mathrm{pH} 4.5$, there was a greater reduction in viable cells than at higher $\mathrm{pH}$ values, thus demonstrating that in normal vaginal $\mathrm{pH}, \mathrm{GBS}$ finds it difficult to grow biofilms. In laboratory settings glucose concentration in the medium was non favourable for biofilm production. GBS showed comparable biofilm formation abilities in all five tested media.

Funding: This research was funded by Research grant, University of Sri Jayewardenepura, Sri Lanka. (Grant no. ASP/01/RE/MED/2017/64)

Acknowledgments: The authors wish to thank the staff members of the Department of Microbiology, Faculty of Medical Sciences, University of Sri Jayewardenepura, Sri Lanka.

Conflicts of Interest: The authors have no conflicts of interest to declare. 


\section{REFERENCES}

1. Edmond KM, Kortsalioudaki C, Scott S, Schrag SJ, Zaidi AK, Cousens S, et al. Group B streptococcal disease in infants aged younger than 3 months: systematic review and meta-analysis. Lancet. 2012 Feb;379(9815):547-56.

2. Dilrukshi GN, Kottahachchi J, Dissanayake DMBT, Pathiraja RP, Karunasingha J, Sampath MKA, et al. Group B Streptococcus colonisation and their antimicrobial susceptibility among pregnant women attending antenatal clinics in tertiary care hospitals in the Western Province of Sri Lanka. J obstet gynaecol. 2020 March;41(1):1-6.

3. Sohail MN, Rathnamma D, Isloor S, Veeregowda B, Sharada R. Detection of biofilm formation ability of Streptococcus agalactiae isolated from bovine mastitis cases. Int. J. Farm Sci. 2019 Jan; 9(1): 107-115.

4. Mah TF, O'Toole GA. Mechanisms of biofilm resistance to antimicrobial agents. Trends Microbiol. 2001 Jan;9(1):349.

5. Borges S, Silva J, Teixeira P. Survival and biofilm formation by Group B streptococci in simulated vaginal fluid at different pHs. Antonie van Leeuwenhoek. 2012 Mar; 101(3):677-82.

6. Ho YR, Li CM, Yu CH, Lin YJ, Wu CM, Harn IC, et al. The enhancement of biofilm formation in Group B streptococcal isolates at vaginal pH. Med Microbiol Immunol. 2013 Apr;202(2):105-15.

7. AL-Kafaween MA, Khan RS, Hilmi ABM, Arrif TM. Characterization of biofilm formation by Escherichia coli: An in vitro study. J App Biol Biotech. 2019 May;7(03):17-9.

8. Borges SF, Silva JGL, Teixeira PCM. Survival and biofilm formation of Listeria monocytogenes in simulated vaginal fluid: influence of pH and strain origin. FEMS Immunol Med Microbiol. 2011 Aug;62(3):315-20.

9. Rosini R, Margarit I. Biofilm formation by Streptococcus agalactiae: influence of environmental conditions and implicated virulence factors. Front Cell Infect Microbiol. 2015 Feb;5:6.

10. Weerasekera MM, Wijesinghe GK, Jayarathna TA, Gunasekara CP, Fernando N, Kottegoda N, et al. Culture media profoundly affect Candida albicans and Candida tropicalis growth, adhesion and biofilm development. Mem Inst Oswaldo Cruz. 2016 Nov;111(11):697-702.

11. Kennedy CA, O'Gara JP. Contribution of culture media and chemical properties of polystyrene tissue culture plates to biofilm development by Staphylococcus aureus. J Med Microbiol 2004;53: 1171-3.

12. Konto-Ghiorghi $Y$, Mairey E, Mallet A, Duménil G, Caliot E, Trieu-Cuot $P$, et al. Dual role for pilus in adherence to epithelial cells and biofilm formation in Streptococcus agalactiae. PLoS Pathog. 2009 May;5(5): e1000422.

13. Rinaudo CD, Rosini R, Galeotti CL, Berti F, Necchi F, Reguzzi V, et al. Specific involvement of pilus type 2a in biofilm formation in group B Streptococcus. PLoS One. 2010 Feb;15;5(2): e9216.

14. Peiris M, Fernando N, Jayaweera P, Weerasekera M, Gunasekara C. Bacteria mediated silver nanoparticles: comparison as potent antibiofilm agents: SLJID.2019 Apr; Vol.9 (1):13-23.

15. Stepanović S, Vuković D, Hola V, Bonaventura GD, Djukic S, Circovic I, et al. Quantification of biofilm in microtiter plates: overview of testing conditions and practical recommendations for assessment of biofilm production by staphylococci. Apmis. 2007; 115(8):891-9.

16. Wijesinghe G, Dilhari A, Gayan B, Kottegoda N, Samaranayake L, Weerasekera M. Influence of Laboratory Culture Media on in vitro Growth, Adhesion, and Biofilm Formation of Pseudomonas aeruginosa and Staphylococcus aureus. Med Princ Pract. 2019 Apr; 28:28-35.

17. Parker RE, Laut C, Gaddy JA, Zadoks RN, Davies HD, Manning SD. Association between genotypic diversity and biofilm production in group B Streptococcus. BMC Microbiology. 2016 May; 16:86.

18. Roesch LFW, Silveira RC, Corso AL, Dobbler PT, Mai V, Rojas BS, et al. Diversity and composition of vaginal microbiota of pregnant women at risk for transmitting Group B Streptococcus treated with intrapartum penicillin. PLOS ONE. 2017 Feb;12(2): e0169916.

19. Ventolini G. Vaginal Lactobacillus: biofilm formation in vivo - clinical implications. Int J Womens Health. 2015 Feb; 7:243-7.

20. Abdul-Lateef LA, Gatea AK, Jwad TS. Biofilm formation by Streptococcus agalactiae is affected by pH changes in vitro: J. Pharm. Sci. \& Res. 2018 Dec;10(12):3216-3218

21. Mu X, Zhao C, Yang J, Wei X, Zhang J, Liang C, et al. Group B Streptococcus colonization induces Prevotella and Megasphaera abundance-featured vaginal microbiome compositional change in non-pregnant women. Peer J. 2019 Aug; 7: e7474.

22. Kaur H, Kumar P, Ray P, Kaur J, Chakraborti A. Biofilm formation in clinical isolates of group B streptococci from north India. Microb Pathog. 2009 Jun; 46:321-7.

23. Patras KA, Nizet V. Group B Streptococcal Maternal Colonization and Neonatal Disease: Molecular Mechanisms and Preventative Approaches. Front Pediatr. 2018 Feb;6:27.

24. Houot L, Watnick PI. A novel role for enzyme I of the Vibrio cholerae phosphoenolpyruvate phosphotransferase system in regulation of growth in a biofilm. J Bacteriol. 2008 Jan;190(1):311-20. 
25. D'Urzo N, Martinelli M, Pezzicoli A, De Cesare V, Pinto V, Margarit I, et al. Acidic $\mathrm{pH}$ strongly enhances in vitro biofilm formation by a subset of hypervirulent ST-17 Streptococcus agalactiae strains. Appl Environ Microbiol. 2014 Apr;80(7):2176-85.

26. Singh AK, Prakash P, Achra A, Singh GP, Das A, Singh RK. Standardization and Classification of In vitro Biofilm Formation by Clinical Isolates of Staphylococcus aureus. J Glob Infect Dis. 2017 Jul;9(3):93-101.

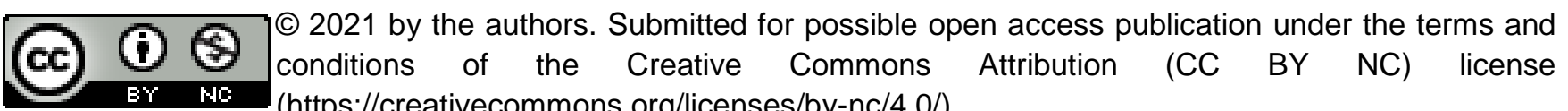
(https://creativecommons.org/licenses/by-nc/4.0/). 